\title{
Ecuaciones diferenciales de la física clásica. Interpretación y solución mediante dinámica de sistemas
}

\section{Differential equations of classical physics. Interpretation and solution through systems dynamics}

\author{
Giampaolo Orlandoni-Merli' ${ }^{1}$ Josefa Ramoni-Perazzi ${ }^{2}$ \\ ${ }^{1}$ Grupo de Investigación en Ciencias Básicas y Aplicadas para la Sostenibilidad - CIBAS, Departamento de Física y Matemática, \\ Facultad de Ciencias Exactas, Físicas y Naturales, Universidad de Santander, Colombia. Email: gorlandoni@udes.edu.co \\ ${ }^{2}$ Grupo de Investigación CIEMPIES, Facultad de Ciencias Económicas, Administrativas y Contables, Universidad de Santander, \\ Colombia. Email: j.ramoni@udes.edu.co
}

RECIBIDO: Marzo 31, 2017. ACEPTADO: Septiembre 10, 2017. Versión FinAL: Octubre 10, 2017.

\begin{abstract}
RESUMEN
Las ecuaciones diferenciales lineales y no lineales constituyen los medios matemáticos para estudiar la dinámica de los sistemas físicos. En general esas ecuaciones pueden ser analizadas y resueltas analíticamente por métodos clásicos. En el caso de sistemas complejos no lineales hay que recurrir a métodos numéricos para su análisis y solución. Utilizando la metodología de Dinámica de Sistemas (DS) es posible representar, analizar y simular el comportamiento de sistemas físicos tanto lineales como no lineales. En este trabajo se estudian algunos modelos físicos mediante DS.
\end{abstract}

PALABRAS CLAVE: Ecuaciones diferenciales; física clásica; dinámica de sistemas.

\begin{abstract}
Linear and nonlinear differential equations are mathematical instruments to study physical systems. In general, these equations can be analyzed and solved analytically by classical methods. In the case of complex nonlinear systems, numerical methods must be used for analysis and solution. Using Systems Dynamics (SD) methodology it is possible to represent, analyze and simulate the behavior of both linear and nonlinear physical systems. In this paper we consider some physical models using SD.
\end{abstract}

KEYWORDS: Differential equations; classic physics; system dynamics.

\section{INTRODUCCIÓN}

Los sistemas físicos usualmente se representan con ecuaciones diferenciales (ED) lineales y no lineales. En general esas ecuaciones pueden ser analizadas y resueltas analíticamente por métodos clásicos. En el caso de sistemas complejos no lineales hay que recurrir a métodos numéricos para su análisis y solución. Por otra parte, la metodología de DS permite modelar estos fenómenos físicos utilizando un lenguaje icónico de programación. La DS permite representar estos fenómenos mediante variables de acumulación y variables de flujo, de manera icónica. Este modelamiento ofrece, a su vez, la posibilidad de resolver dichas ecuaciones por los métodos numéricos tradicionales (Euler, Runge-Kutta fijo, Runge-Kutta variable). 
Además de generar soluciones, la DS permite hacer simulación de los sistemas modelados, mediante variación de los parámetros que definen dichos sistemas, obteniéndose así los comportamientos dinámicos de las variables de estado involucradas [1], [4], [6], [11].

En este marco de referencia se formulan, analizan y simulan algunos modelos de física clásica utilizando las herramientas de la DS. Son objetivos de este trabajo presentar las ecuaciones diferenciales asociadas a modelos clásicos de la física. Mediante la metodología de DS y, basados en los modelos planteados, se construyen modelos dinámicos para así simular el comportamiento de los sistemas considerados.

\section{MÉTODOS}

\subsection{Representación y solución de modelos físicos mediante dinámica de sistemas}

La DS es una metodología de análisis que estudia de qué manera la estructura de retroalimentación de un sistema genera su comportamiento dinámico; es decir, trata de describir las fuerzas que surgen internamente en el sistema analizado para generar los cambios que en él se producen a lo largo del tiempo, y de qué forma se interrelacionan dichas fuerzas [1]. La DS construye modelos dinámicos para predecir efectos a largo plazo de decisiones alternativas: a) Observa el comportamiento del sistema real, identificando sus elementos fundamentales, b) Busca en el sistema las estructuras de retroalimentación que puedan explicar su comportamiento observado, c) Construye modelos matemáticos del comportamiento del sistema para poderlo simular en un computador, d) Valida, modifica y analiza el modelo, ante diferentes escenarios. Los análisis de DS se caracterizan por estudiar el sistema en forma continua, considerándose así los eventos de manera agregada. La DS se concentra en las tasas de cambio de las diferentes magnitudes o niveles que intervienen en el ecosistema estudiado [1], [4], [11].

\subsubsection{Modelaje mediante dinámica de sistemas.}

El proceso de modelaje según el enfoque de DS, se centra en la visión global (global view) del problema en estudio, progresando desde la conceptualización y llegando a los detalles de formulación de ecuaciones y pruebas del modelo. La esencia de la visión global se basa en dos supuestos interrelacionados: la búsqueda de objetivos del sistema (goal-seeking), y la estructura de retroalimentación del sistema (feedback structure). La búsqueda de objetivos requiere la existencia de la estructura de retroalimentación, que es un proceso circular, en el cual cualquier desviación de las condiciones actuales respecto de las condiciones deseadas estimula acciones para que tales condiciones regresen al estado deseado. Este punto de vista se denomina estructura interna de retroalimentación y conlleva la idea de que el comportamiento del sistema no es producto de impactos externos, sino de la forma como la estructura de retroalimentación del sistema procesa esos impactos [1], [4], [6].

Esto implica, a su vez, considerar dos nuevos enfoques:

- Cambio de modelaje discreto a modelaje continuo: los lazos de retroalimentación y las actividades de búsqueda de objetivos son continuos en su operación.

- Cambio de la perspectiva de equilibrio estático a desequilibrio dinámico: la causalidad no es un concepto lineal sino circular, lo cual lleva a la idea del comportamiento desequilibrado del sistema. Si el sistema está en equilibrio no hay discrepancia entre el nivel actual y el deseado, por lo que no hay cambios, mientras que la búsqueda de objetivos implica un cambio constante.

Los aspectos principales del enfoque de DS son:

a) Determinar los limites apropiados para decidir los elementos que se van a incluir en el sistema en estudio,

b) Pensar en términos de relaciones tipo causaefecto $y$

c) Centrarse en las relaciones de retroalimentación entre los componentes del sistema.

Una de las ideas fundamentales en el enfoque de DS es considerar la estructura del sistema como una red causal de lazos de retroalimentación (feedback loops), que explica el comportamiento de los elementos del sistema a lo largo del tiempo.

\subsubsection{Lazos de retroalimentación en dinámica de sistemas.}

Existen dos tipos de lazos de retroalimentación en DS:

a) Retroalimentación positiva: si una magnitud comienza a crecer, se produce un efecto "bola de nieve", es decir, esa magnitud sigue creciendo, generalmente, a una tasa más rápida. Un crecimiento exponencial se produce, por ejemplo, si al aumentar la población total, aumenta el número de nacimientos, esto produce un aumento mayor de la población y, a su vez, un aumento, aun mayor, en el número de nacimientos.

b) Retroalimentación negativa: los lazos de RN tienden a mantener el sistema bajo control, produciendo comportamientos estables debido al principio de autorregulación. El equilibrio en 
un lazo negativo es estable: el sistema tiende al equilibrio luego de producirse perturbaciones. Por ejemplo, el aumento en la población total trae como consecuencia un incremento de la mortalidad; esto lleva a una disminución en el nivel de la población, que, con cierto retardo produce una disminución en la mortalidad. Esto se traduce en un posterior aumento poblacional, que conlleva a un nuevo aumento en la mortalidad.

\subsection{Componentes básicos de un sistema según el enfoque de dinámica de sistemas}

Los modelos de DS se componen básicamente de variables y ecuaciones de nivel y de flujo.

\subsubsection{Las variables de nivel y variables de flujo.}

a) Variables de nivel o de estado. Los niveles representan la acumulación de recursos en el sistema a lo largo del tiempo, como resultado de la diferencia acumulada entre los flujos de entrada y los de salida para dicho nivel. Representan el estado del sistema en un momento dado.

b) Variables de flujo. Estas representan el flujo de material o energía desde o hacia un cierto nivel, determinando los cambios en dichas variables por unidad de tiempo.

\subsubsection{Ecuaciones de nivel y de flujo.}

a) Ecuaciones de nivel: son las ecuaciones integrales del sistema; relacionan una magnitud en el tiempo $\mathbf{t}$ con su valor en el periodo anterior (t-1), y con su tasa de cambio, en el intervalo de cálculo dt. La expresión general es Nivel $_{t}=$ Nivel $_{t-1}+\boldsymbol{d} \boldsymbol{t}$ Tasa $_{(t-1 ; t)}$. Para el ejemplo del crecimiento poblacional la ecuación se expresa: $\boldsymbol{P o b}_{t}=\boldsymbol{P o b}_{t-1}+\operatorname{dt}(\text { Nat }- \text { Mort })_{(t-1 ; t)}$.

b) Ecuaciones de flujo: determinan el comportamiento del sistema y representan la

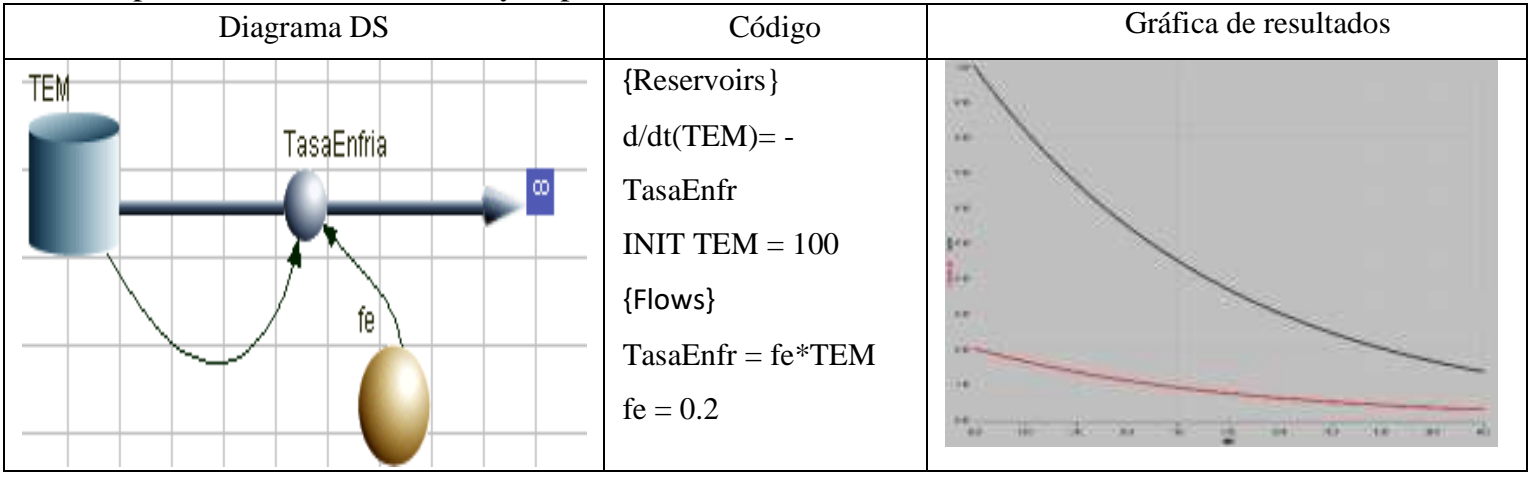

Figura 1. Ecuación diferencial de primer orden. Diagrama DS Madonna Berkeley, 2017. Fuente: Elaboración propia. tasa de variación de las variables nivel en cada unidad de tiempo.

\subsection{Representación de los modelos de dinámica de sistemas mediante íconos}

\subsubsection{Símil hidráulico de la ecuación fundamental de dinámica de sistemas.}

La ecuación diferencial básica de DS pude ilustrarse mediante un símil hidráulico: un tanque de agua (nivel $\mathbf{N}$ ), que se llena según un flujo de entrada $\mathbf{E}$, y se vacía según un flujo de salida $\mathbf{S}$. La ecuación que relaciona el nivel de agua del tanque con los flujos de entrada y de salida se expresa de esta manera: $\mathbf{d N} / \boldsymbol{d} \mathbf{t}=\mathbf{N}^{\prime}=(\mathbf{E}-\mathbf{S})$. $\mathrm{Si}$ la salida es proporcional al nivel de agua existente en el tanque, el flujo de salida se expresa como $\boldsymbol{S}=\boldsymbol{K} \boldsymbol{N}$. Entonces la derivada del flujo salida $\boldsymbol{S}^{\boldsymbol{\prime}}=\boldsymbol{K} \boldsymbol{N}^{\boldsymbol{\prime}}=\boldsymbol{K}(\boldsymbol{E}-\boldsymbol{S})$. En forma de ecuación en diferencias se expresa $\boldsymbol{S}_{t+d t}=\boldsymbol{S}_{t}+\boldsymbol{K}(\boldsymbol{E}-\boldsymbol{S}) \boldsymbol{d t}$. La ecuación anterior indica que un aumento brusco en la entrada, hace que la salida se vaya ajustando en una fracción (Kdt) del flujo neto (E-S). La constante $K=(\mathbf{1} / \mathbf{R})$ representa la fracción de ajuste del sistema, indicando que a menor retardo $\mathrm{R}$, mayor es la fracción de ajuste, y más rápido se adapta la salida a la entrada del sistema [1], [4].

\subsubsection{Representación de ecuaciones diferenciales con iconos de dinámica de sistemas.}

Para representar una ecuación diferencial mediante un diagrama de DS debe recordarse que una variable de flujo equivale a una derivada temporal, y que una variable de nivel o estado es una acumulación o integral de los flujos durante un periodo de tiempo dt. Si la variable de nivel se denota por TEM, y la variable de flujo por TasaEnfria, entonces la figura 1 muestra la pérdida de temperatura de un cuerpo a una fracción fe de su temperatura TEM. 


\section{APLICACIONES}

\subsection{Ley de Newton de Enfriamiento/ Calentamiento}

La velocidad a la cual la temperatura de un cuerpo cambia es proporcional a la diferencia entre la temperatura del cuerpo (TEM) y la temperatura del medio circundante $(\mathrm{Tm})$. Si dTEM/dt es la velocidad a la cual la temperatura del cuerpo cambia, entonces $\boldsymbol{d T E M} / \boldsymbol{d} \boldsymbol{t}=\boldsymbol{k}(\boldsymbol{T}-\boldsymbol{T m})$, donde $\boldsymbol{k}$ es una constante de proporcionalidad [7].

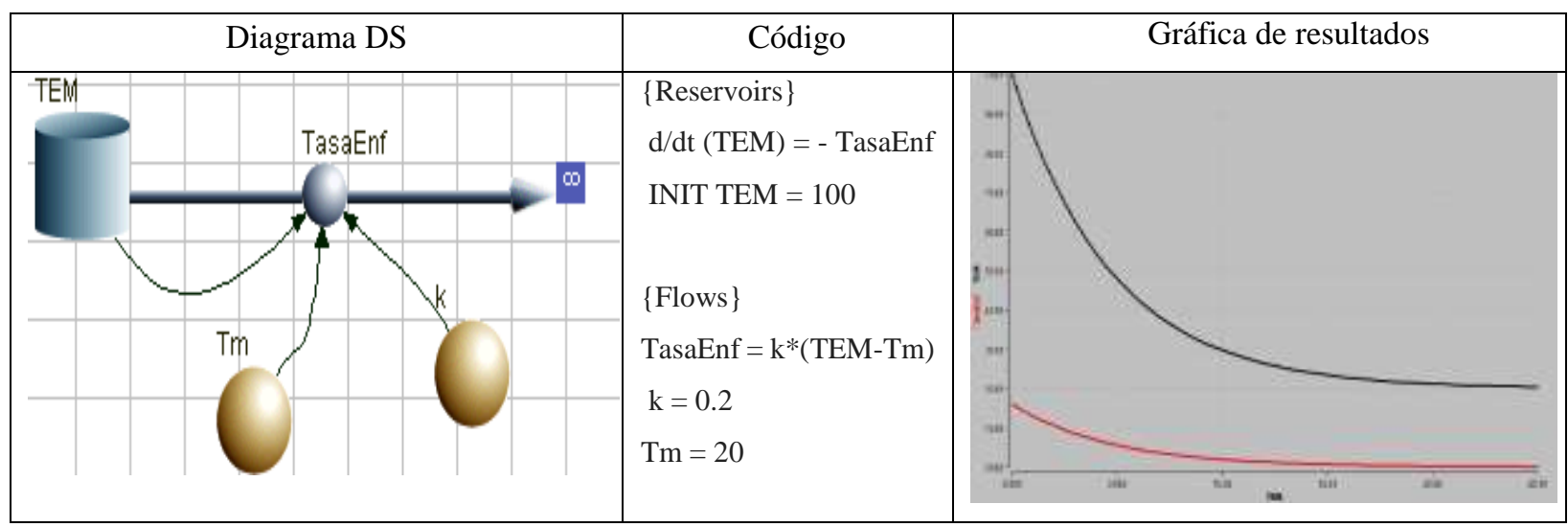

Figura 2. Ecuación diferencial de primer orden. Enfriamiento con objetivo. Diagrama y código ds madonna berkeley. Fuente: Elaboración propia.

\subsection{Ley de Newton y el sistema masa-resorte}

Se cuelga una masa $m$ de un resorte, se estira en determinada longitud, y si la masa se desplaza en una cantidad X desde su posición de equilibrio, la aplicación de la segunda ley de Newton genera la siguiente ecuación diferencial de segundo orden:

$$
\frac{\mathrm{d}^{2} \mathrm{X}}{\mathrm{dt}^{2}}+\left(\frac{\mathrm{k}}{\mathrm{m}}\right) \mathbf{X}=\mathbf{0}
$$

La ecuación 1 es una ecuación diferencial de segundo orden no lineal y puede expresarse como un sistema de dos ecuaciones diferenciales lineales de primer orden. En la figura 3 se representa mediante gráfico de DS, y se muestran los resultados de la corrida para los parámetros indicados. El código usado es Madonna.

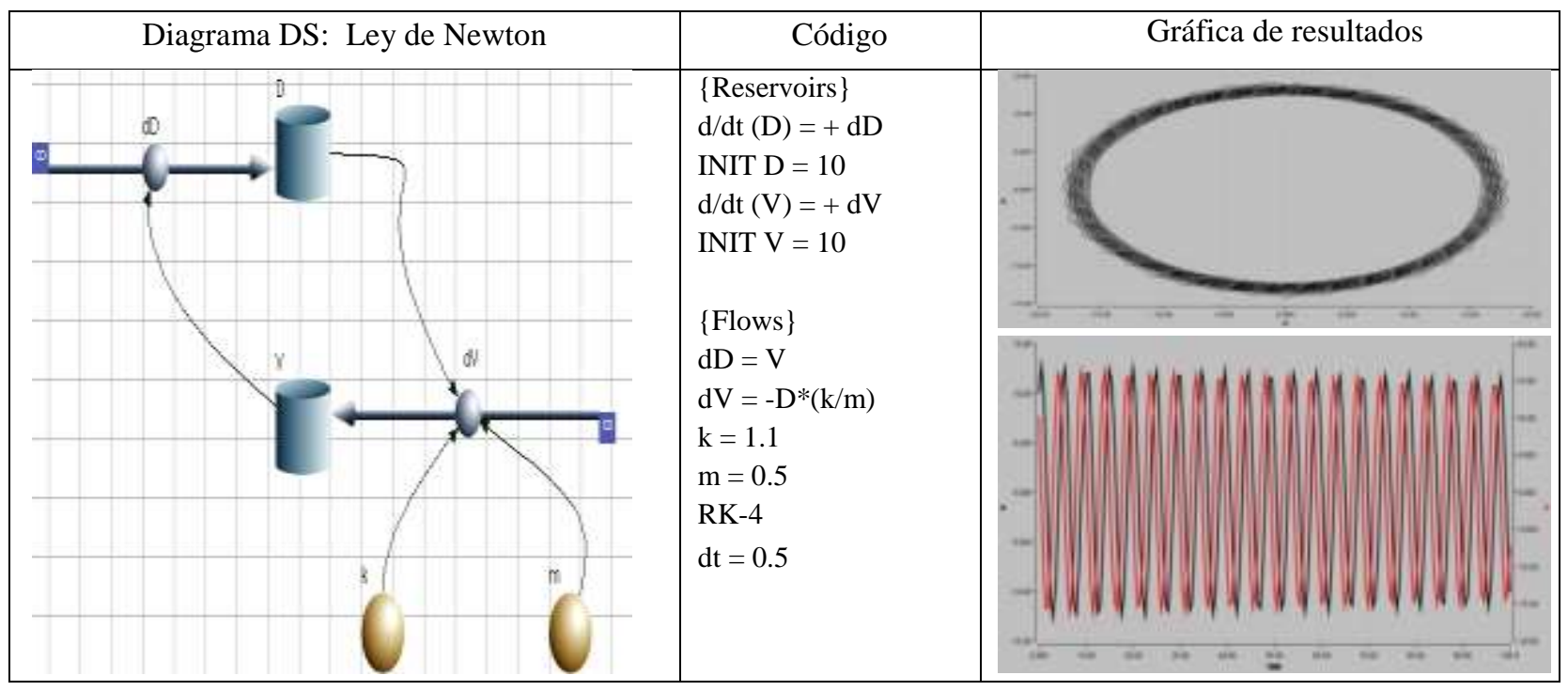

Figura 3. Ecuación Diferencial de Segundo Orden. Sistema Masa-Resorte. Diagrama y Código DS Madonna Berkeley. Fuente: Elaboración propia. 


\subsection{Modelo termostato}

El control termostático de cierto ambiente es un ejemplo de un sistema mecánico simple, que se caracteriza por oscilar. El propósito de un termostato es mantener una sala en un rango determinado de temperatura, sin necesidad de encender y apagar constantemente el horno conectado a dicho instrumento; para ello, el control debe establecer un intervalo de temperatura. El horno se enciende cuando la temperatura desciende por debajo del límite inferior, y cuando sube por encima del límite superior de temperatura, el horno se apaga. Para todas las temperaturas dentro del rango, el horno permanece encendido si está encendido, y permanece apagado si está apagado. Este modelo considera la presencia de un evento discreto (encender o apagar el horno), y la conmutación del horno depende de si el horno ya está encendido. La figura 4 muestra el diagrama de DS y los resultados de una corrida para los parámetros indicados, usando el código Vensim PLE.

\subsection{Modelos de ecuaciones diferenciales no lineales de sistemas caóticos}

Los modelos de ecuaciones diferenciales no lineales de tercer orden generalmente producen resultados caóticos. Entre los más conocidos está el modelo de Lorenz y el modelo de Rossler. A continuación se desarrollan dichos modelos en el entorno del enfoque de Dinámica de Sistemas.

\subsubsection{Modelo de caos de Lorenz.}

Si se hierve agua en un recipiente, las partículas próximas a la fuente de calor tienden a subir, generándose un movimiento circular o convección si la diferencia de temperatura es considerable. Basado en esta idea, el meteorólogo Edward Lorenz en 1963 definió un sistema dinámico determinista no lineal tridimensional conformado por tres ecuaciones diferenciales que incorporan dos no linealidades (x.y; x.z), y que permite

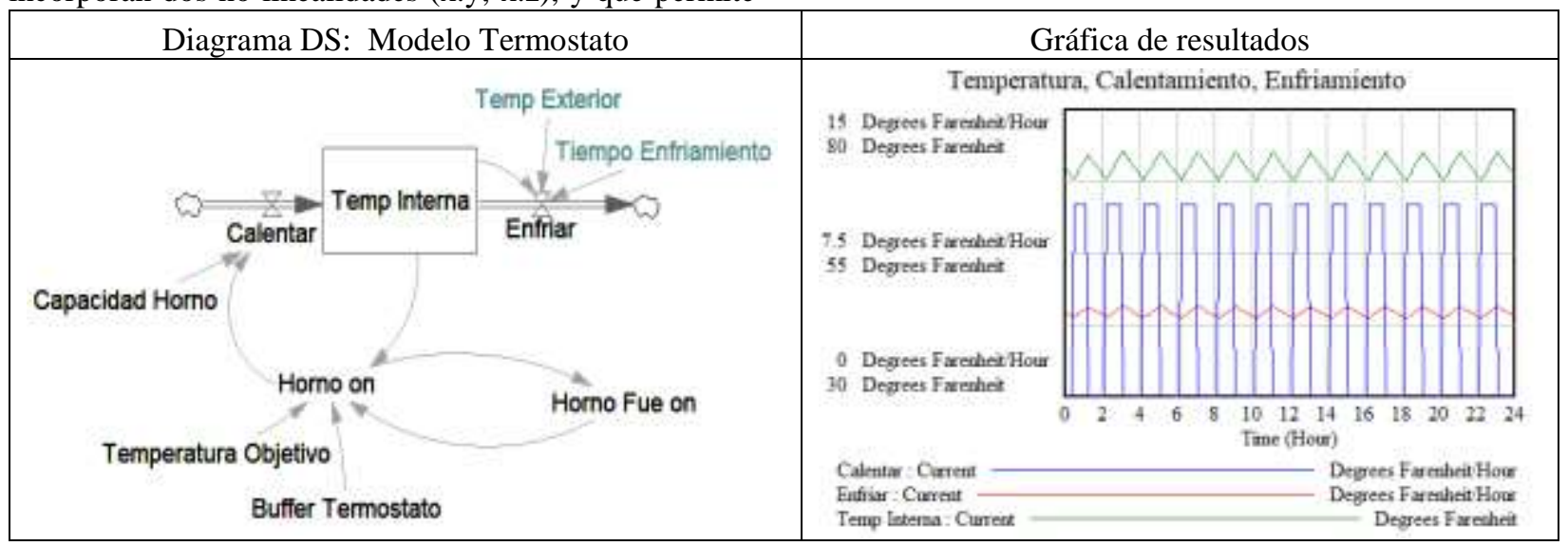

modelar el comportamiento meteorológico en la atmósfera [3]. El modelo se define con tres variables de nivel (flujo convectivo; diferencia de temperatura horizontal; diferencia de temperatura vertical), tres variables de flujo (x:variación de flujo convectivo, y:variación de temperatura horizontal, z:variación de temperatura vertical), y tres parámetros adimensionales: el número de Prandtl $(\sigma)$, que establece una relación entre la viscosidad y la conductividad térmica del fluido; el número de Raleigh $(\rho)$, que cuantifica la transmisión de calor en una capa de fluido a través de la radiación térmica interna, y la altura $(\beta)$, que representa el espesor de la capa que se está estudiando. El sistema de las tres ecuaciones diferenciales no lineales de Lorenz se muestra a continuación:

El artículo debe explicar claramente los procedimientos utilizados para el desarrollo de la investigación y la obtención de los resultados presentados. Se debe presentar el objeto, mencionar las circunstancias en que se realizó el estudio y la manera en que este se estructuró.

$$
\begin{aligned}
& \dot{x}=\sigma \mathbf{y}-\sigma \mathbf{x} \\
& \dot{y}=\rho \mathbf{x}-\mathbf{y}-\mathbf{x y} y \\
& \dot{z}=\mathbf{x} \mathbf{y}-\beta \mathbf{z}
\end{aligned}
$$

El diagrama de DS de la figura 5 muestra gráficamente dicho sistema de tres ecuaciones. La simulación mediante DS del sistema definido, para los valores especificados de los parámetros (sigma $=10$, rho $=28$, beta $=2,67$; $\mathrm{x} \_$init $=1 ; \quad \mathrm{y} \_$init $=0,5 ; \quad \mathrm{z} \_$init $=0,1 ;$ delta_t $=0,001725$; RK4_variable) genera las gráficas del efecto mariposa que se muestran en el espacio de fases de la figura 6, donde cada punto representa un posible estado del sistema.

Figura 4. Sistema Termostato. Diagrama y Código Vensim PLE. Fuente: Elaboración propia. 


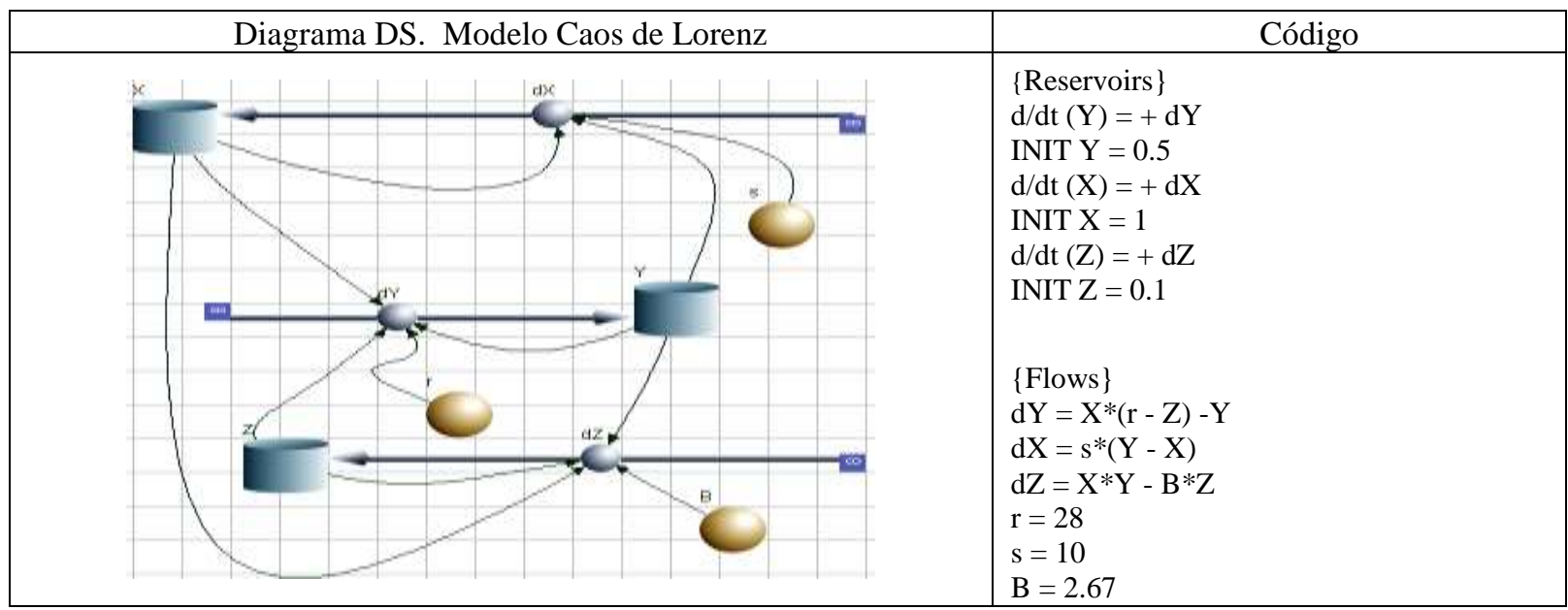

Figura 5. Sistema Caos Lorenz. Diagrama y Código DS Madonna Berkeley. RK4; dt =0.0125. Fuente: Elaboración propia.

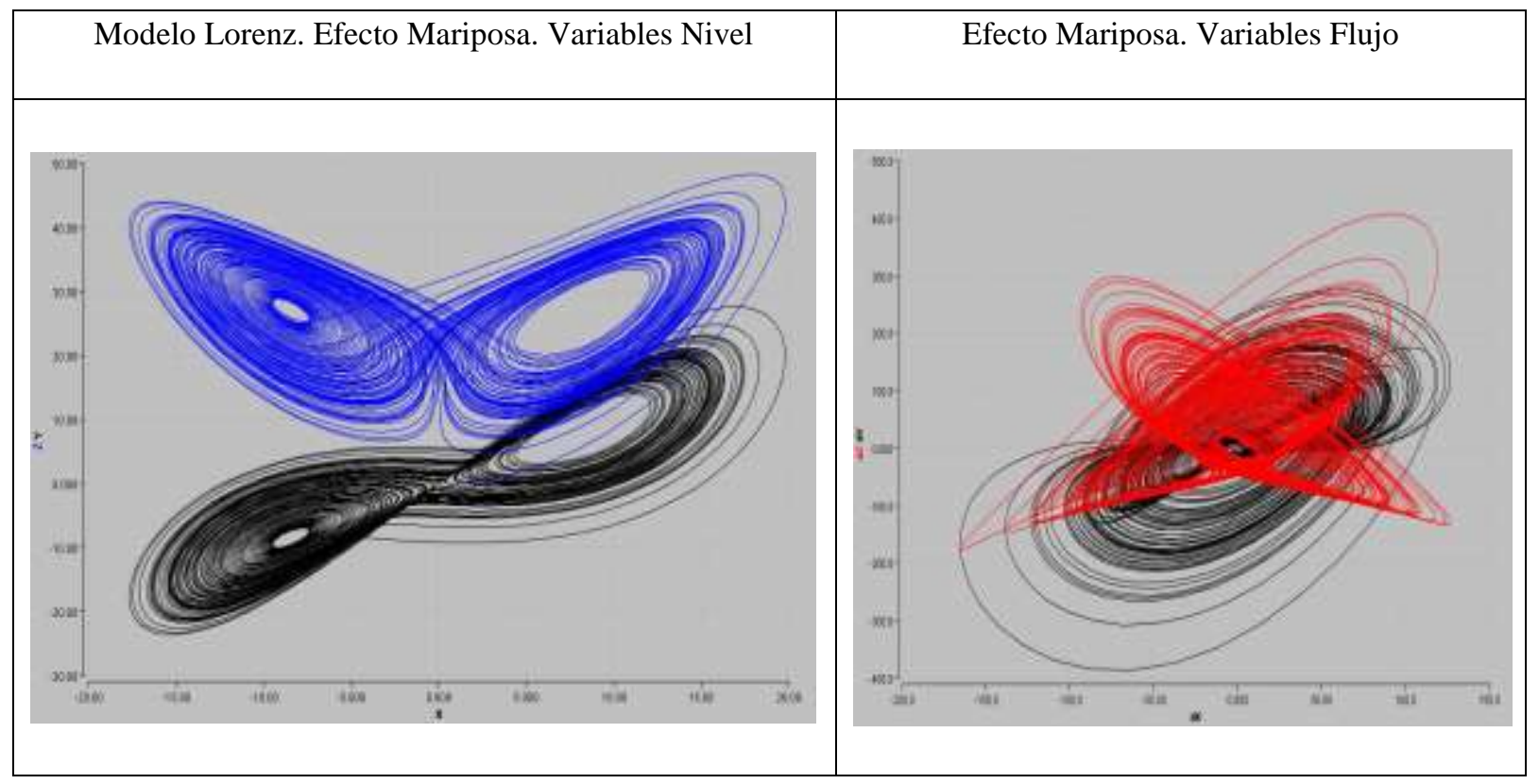

Figura 6. Gráficos Efecto Mariposa. Resultados de simular el sistema Caos de Lorenz. Fuente: Elaboración propia.

Las gráficas del efecto mariposa para las variables de nivel y las variables de flujo se muestran en el espacio de fases de la figura 6 , donde cada punto representa un posible estado del sistema.

\subsubsection{Modelo de caos de Rossler.}

El sistema caótico de Rossler, versión simplificada de las ecuaciones de flujo no periódico determinístico de Lorenz, es un sistema dinámico de tercer orden y se expresa en las tres ecuaciones diferenciales siguientes:

$$
\begin{aligned}
& \dot{x}=-(y+z) \\
& \dot{y}=\mathbf{x}+\mathbf{a y} \\
& \dot{z}=\mathbf{b}+\mathbf{x z}-\mathbf{c z}
\end{aligned}
$$

El diagrama de DS del modelo y los resultados de algunas corridas de simulación se muestran en la figura 7, para las condiciones iniciales de las variables: [ $\mathrm{Xo}=1 ; \mathrm{Yo}=1$; $\mathrm{Zo}=1]$; y los valores de los parámetros: $[\mathrm{a}=0.25 ; \mathrm{b}=2.00$; $\mathrm{c}=4.00]$. 


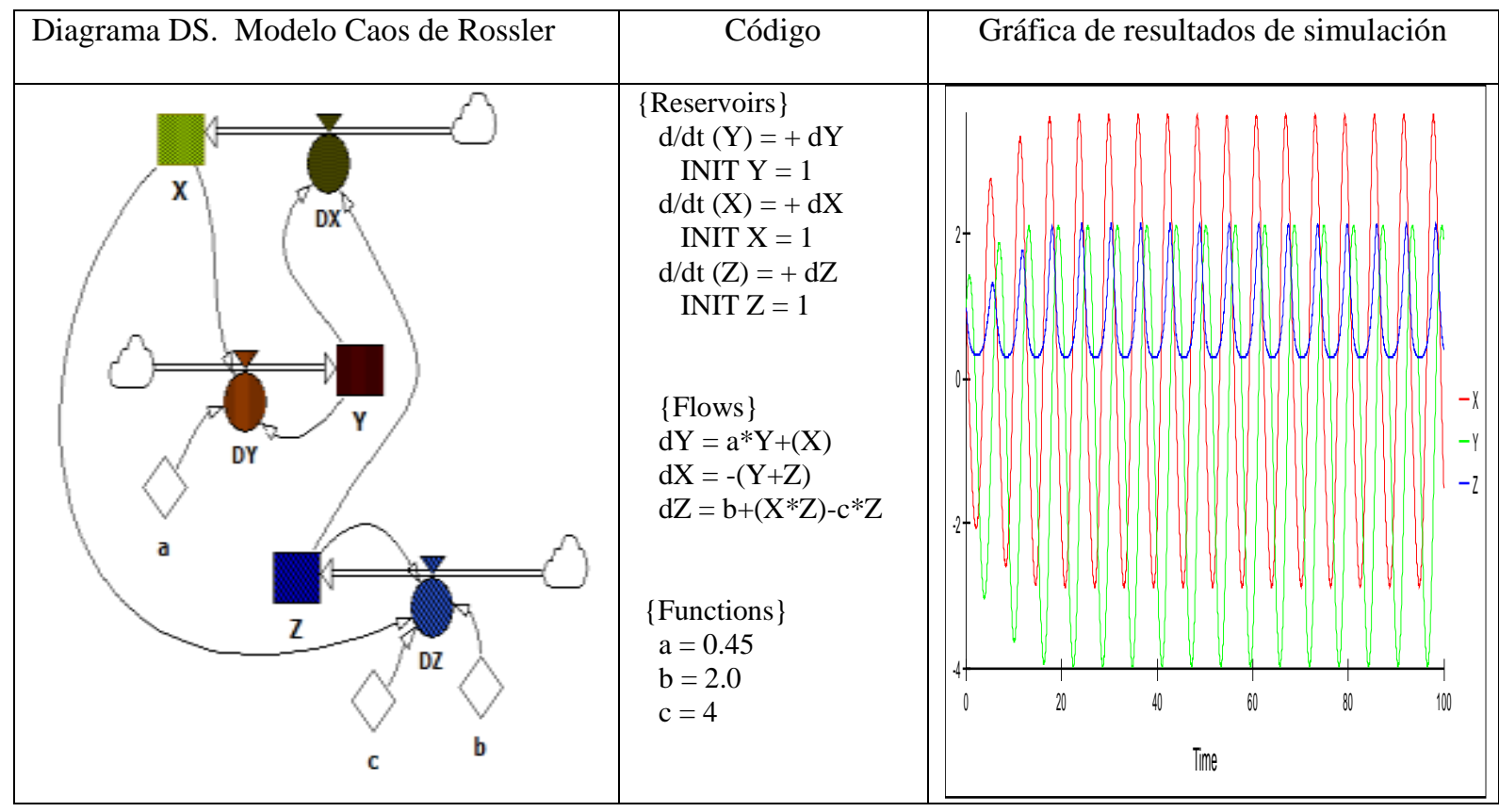

Figura 7. Sistema Caos Rossler. Diagrama y Código DS Powersim. Parámetros: $a=0.25 ; b=2.00 ; c=4.00$. Fuente: Elaboración propia.

La figura 8 muestra otros diagramas de fases, que resultan de las corridas de simulación realizadas:

El análisis de este sistema para las condiciones iniciales $\left[\mathrm{Xo}=1 ; \mathrm{Yo}_{\mathrm{o}}=1 ; \mathrm{Zo}=1\right]$, y los parámetros $[\mathrm{b}=2.00 ; \mathrm{c}=$ 4.00] indica que:

- Para $\mathrm{a}=0.25$, la simulación del modelo produce un equilibrio cíclico: las series muestran oscilaciones cíclicas regulares. El atractor correspondiente es una curva cerrada única.
- Para a=0.45, el comportamiento dinámico del sistema se hace caótico; las series no tienen un patrón discernible: es una mezcla de oscilaciones de diferentes amplitudes que ocurren sin ningún orden ni frecuencia. El atractor es caótico y representa un camino que nunca se repite (gráficamente nunca se cruza). Se genera un fractal (atractor extraño), verificando que el orden y la aleatoriedad pueden coexistir simultáneamente. En la figura 9 se grafican algunos resultados de las simulaciones realizadas con estos valores de parámetros.
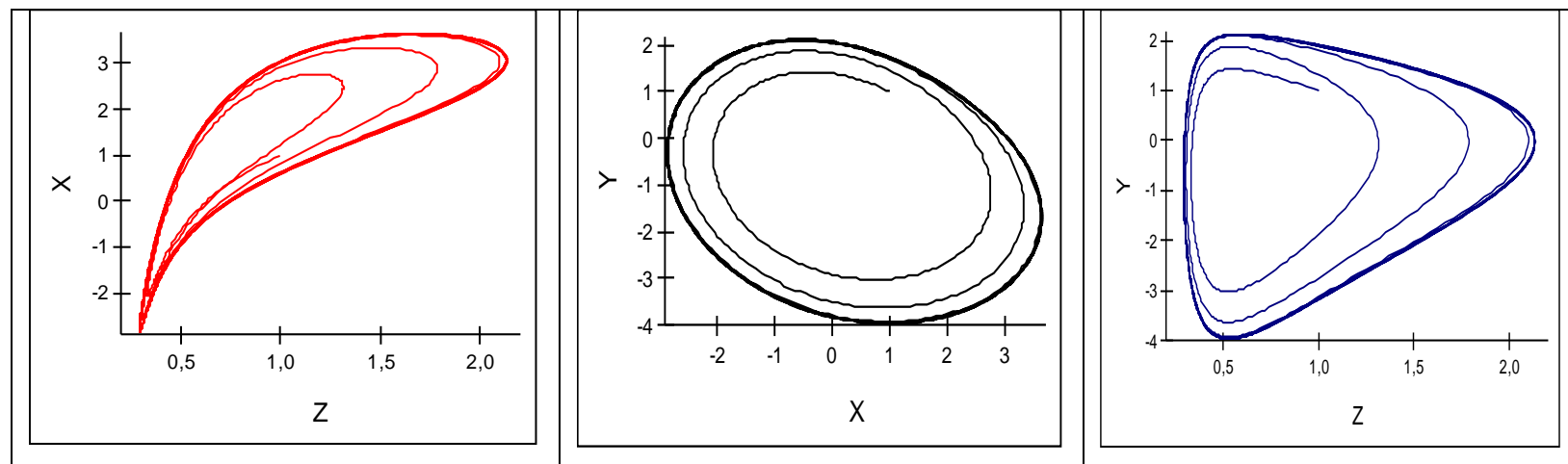

Figura 8. Sistema Caos Rossler. Gráficos de Simulación. Parámetros: a = 0.25; b = 2.00; c = 4.00. Fuente: elaboración propia.

\section{CONCLUSIONES}

La DS es a la vez una filosofía de análisis de sistemas y una herramienta eficiente para representar y estudiar sistemas dinámicos complejos a través de ecuaciones diferenciales ordinarias (EDO). La DS permite además estudiar características específicas a través del mecanismo de la simulación, que puede ejecutarse con software pertinente, como por ejemplo Powersim, Vensim, Madonna, entre otros. 

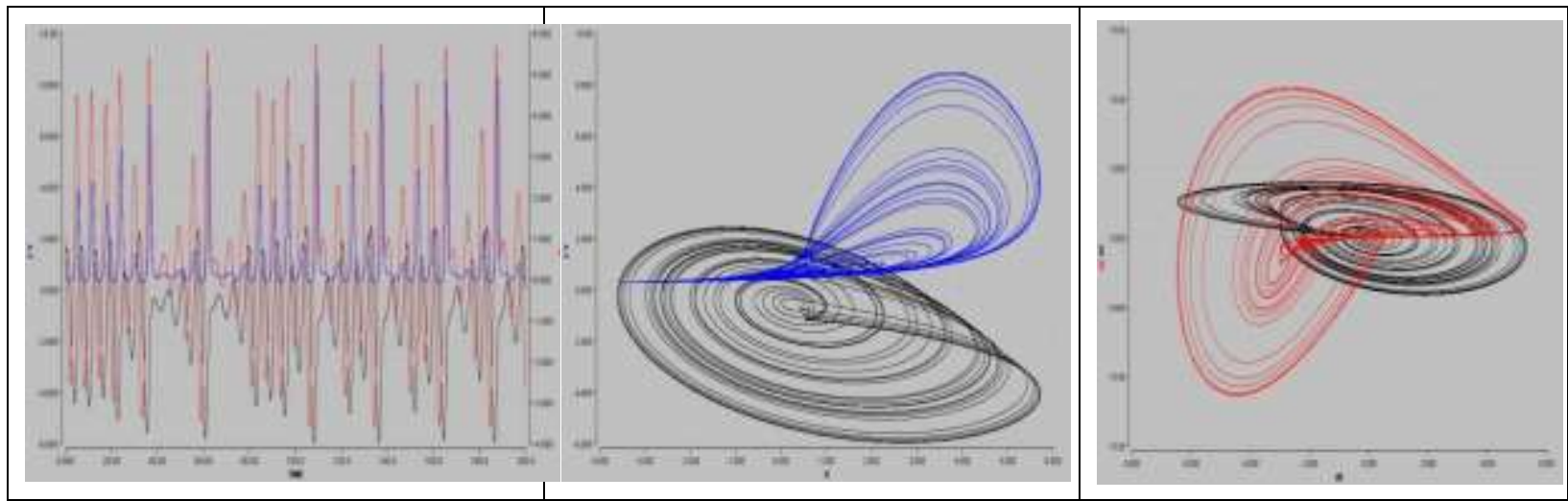

Figura 9. Sistema Caos Rossler. Gráficos de Simulación. Parámetros: $a=0.45 ; b=2.00 ; c=4.00$. Fuente: elaboración propia.

En este trabajo se han presentado algunos modelos de la física clásica bajo el enfoque de DS, ilustrando su utilidad en la representación, análisis y simulación de esos sistemas físicos. La DS permite representar, resolver y simular EDO de cualquier orden, lo que la hace una herramienta muy útil en la enseñanza de la física, cuyos modelos y estructuras naturalmente se expresan como ecuaciones diferenciales. Son muchas las ventajas en este sentido, destacándose: 1) la DS permite visibilizar los conceptos abstractos de la física, y comprender sus interrelaciones mediante su representación gráfica con variables de nivel o estado y variables de flujo, utilizando un lenguaje icónico-gráfico; 2) Las soluciones de los sistemas de EDO que representan los modelos físicos teóricos se obtienen de forma automática, utilizando diferentes algoritmos numéricos, como los métodos de Euler y Runge Kutta de paso fijo y variable. Además es inmediato el cambio en el intervalo de tiempo dt, utilizado en la solución de las ecuaciones, lo que permite ver su efecto en la precisión de los resultados obtenidos de forma gráfica y numérica, produciéndose diagramas de fases y tablas y gráficos dinámicos; 3)Una gran ventaja en el estudio de modelos físicos con DS es la posibilidad de modificar fácilmente los supuestos que subyacen en los modelos, además de poder introducir relaciones entre variables que incorporan no linealidades de difícil solución con los procedimientos tradicionales. Los softwares disponibles permiten intercambiar el lenguaje gráfico-icónico con el lenguaje matemático de manera inmediata, lo que ayuda a asociar los conceptos abstracto-matemáticos con los esquemas gráficomentales; 4) Finalmente, tal vez la ventaja mayor reside en la factibilidad de hacer análisis de sensibilidad y simular el sistema físico modelado en DS, variando las condiciones iniciales (valores iniciales de las variables de nivel y valores de los parámetros del sistema). Esto permite ir más allá de las soluciones clásicas y abre el espacio a caminos insospechables sin este enfoque, como es la generación de patrones caóticos, difícilmente abordables por los métodos clásicos, y que son típicos de las EDO no lineales, que representan situaciones mucho más acordes con el mundo real en estudio que las ecuaciones lineales.

\section{REFERENCIAS}

[1] I. Forrester, "Principle of Systems, Lexington," USA: Mass, 1961.

[2] R.M. May, "Simple Mathematical Models with very Complicated Dynamics," Nature, vol. 261, pp. 459-467, 1976.

[3] H.W. Lorenz, "Nonlinear Dynamical Economics and Chaotic Motion”, 2 ${ }^{\text {nd }}$ ed, Springer-Verlag, 1993.

[4] G. Orlandoni, "Análisis Dinámico de Poblaciones Biológicas mediante Dinámica de Sistemas," Economía, no. 13, pp. 115-146, Universidad de Los Andes, 1997.

[5] G. Richardson, "Loop polarity, loop dominance, and the concept of dominant polarity," System Dynamics Review, vol. 11, 1984. Reprinted, John Wiley, 1995.

[6] J. Sterman, "Business Dynamics: Systems Thinking and Modeling for a Complex World," McGraw Hill, 2000 .

[7] D. Zill, "A first course in Differential Equations, with modeling applications," $10^{\text {th }}$ ed, Cengage, 2013.

[8]Vensim, Ventana System,PLE. 2017.

[9]R. Macey y G. Oster, Berkeley Madonna, University of Berkeley, 2017

[10]Powersim, Powersim Studio 10, 2017

[11] H. Andrade y X. M. Navas, "Ingeniería de sistemas - Realidad Virtual y Aprendizaje," Rev. UIS Ing., vol. 1, no. 1, pp. 3-9, 2002. 